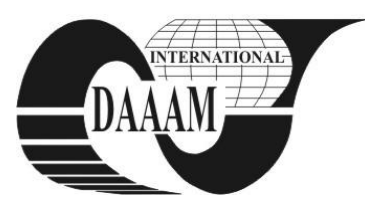

\title{
ADAPTIVE CONTROL OF A THREE-LINKMANIPULATOR AT END-EFFECTOR MOVEMENT ON OPTIMAL TRAJECTORIES
}

\author{
POLIAKOV, A[leksandr] M[ykhaylovich]; KOLESOVA, M[arina] \& CHEPENYUK, O[lena]
}

\begin{abstract}
In this paper we present a solution of the adaptive control problem of the manipulator of robot Mitsubishi $R V-1 A$ at the end-effector motion on the optimal (in terms of a set of criteria) trajectories. For the trajectories planning a minimal torque changes criteria and a minimal discomfort criteria, founded with a biomechanical point of view was used. Control system that uses a linearized manipulator model is designed on the basis of the method proposed by H. Seraji. The law change of error vector of the trajectory tracking and the vector of torques which realize the trajectory close to optimal were obtained. A program that allows to calculate the points coordinates of the optimal trajectory in the manipulator workspace and realizes this control system can be used at the control of various mechanical devices with articulated rigid links and, in particular, in the control of prosthetic limbs with a heterogeneous knee mechanism.
\end{abstract}

Key words: manipulator, quality criterion, optimal trajectory, adaptive control system

\section{INTRODUCTION}

Trajectories planning of the robot arm end-effector - one of the most important tasks of robotics. Its solution is necessary, above all, to manipulator control in the performance of typical or atypical features. In fact, the problem of the manipulator control is divided into two subtasks, one of which is the task of the trajectories planning.

There are many concepts of the trajectories planning, based on kinematic or dynamic principles. However, the most interesting among them is the concept of optimal planning. Thus, in manipulator workspace are searching for the trajectories which are optimal in terms of various quality criteria (LaValle, 2006).

Among the set of optimal planning criteria we shall distinguish those justified from a biomechanical point of view, for example: minimum jerk, minimum torque change, minimum metabolic energy and others (Engelbrecht, 2001). Since the manipulators are devices that replicate the movements of the person's hand, the use for their trajectory planning biomechanical criteria contributes to reproduce the end-effector movements that are similar to movements of the hands. From many points of view, such movement can be considered optimal.

For human hands trajectory planning in a horizontal plane Y. Uno and colleagues proposed a minimum torque change model (Uno et al., 1989) and later - a minimum commanded torque change model (Nakano et al., 1999). F. Crevecoeur with colleagues has applied these models to study the human arm movements in vertical plane (Crevecouer, et al., 2009).

Use of criteria, grounded in terms of these models allows generating a trajectory characterized by the parameters observed in the free movements of human hand. However, with relation to the redundant manipulator the solution of this problem turns out to be ambiguous, because these criteria do not allow choosing the formal optimal boundary conditions that characterize the trajectory. This contradiction can be resolved by introducing an additional criterion, such as prohibiting the arm to accept the boundary configuration, or close to it.
In this paper, for the end-effector trajectories planning in the workspace of three-link manipulator of robot Mitsubishi $\mathrm{RV}-1 \mathrm{~A}$ a set of criteria was used: minimum torque change and minimal discomfort (Marler et al., 2005). Such trajectories were considered later as etalon.

Synthesis of control system for realizing the etalon trajectories is based on the method proposed by $\mathrm{H}$. Seraji, which distinguishing feature is the use of the manipulator inversion in feedforward controller.

The main objective of work is to study the possibility of practical realization of the trajectories observable in human hand movements at the end-effector motion in the workspace of redundant anthropomorphic manipulator.

\section{TRAJECTORIES PLANNING}

The task of the end-effector trajectory planning at motion in sagittal plane is considered on the example of robot Mitsubishi RV-1A.To solve this task we used the dynamic equation of the manipulator of robot with rotational degrees of freedom, which in the general case is as follows:

$$
M(\Phi) \ddot{\Phi}+N(\Phi, \dot{\Phi})+H(\dot{\Phi})=T(t)
$$

where $\Phi, \dot{\Phi}, \ddot{\Phi}, T(t)$ - $n$-dimensional column vectors of generalized coordinates $q_{i}$, velocities $\dot{q}_{i}$, accelerations $\ddot{q}_{i}$ and torques $\tau_{i}$ in the links joints, respectively; $M(\Phi)$ - positive definite symmetric inertia matrix, size $n \times n ; N(\Phi, \dot{\Phi}), H(\dot{\Phi})-$ $n$-dimensional column vectors: the moments caused by the Coriolis, centrifugal, gravitational forces and friction, respectively. Using the criterion of minimum change of torque

$$
K_{1}=\frac{1}{2} \int_{0}^{t_{k}} \sum_{i=1}^{n}\left(\frac{d \tau_{i}}{d t}\right)^{2} d t \rightarrow \min ,
$$

we obtain a functional

$$
\left.I=\frac{1}{2} \int_{0}^{t_{k}} F\left(t, q_{i}, \dot{q}_{i}, \ddot{q}_{i}, \dddot{q}_{i}\right)\right) d t, i=1, . ., n .
$$

A necessary condition of its extremum is the system of EulerPoisson equations:

$$
\frac{\partial F}{\partial q_{i}}-\frac{d}{d t} \frac{\partial F}{\partial \dot{q}_{1}}+\frac{d^{2}}{d t^{2}} \frac{\partial F}{\partial \ddot{q}_{i}}-\frac{d^{3}}{d t^{3}} \frac{\partial F}{\partial \dddot{q}_{i}}=0, i=1, . ., n,
$$

which solution, in turn, is a column vector of generalized coordinates $\Phi(t)$ as a function of time, which uniquely determines the desired trajectory of the manipulator end-effector. The boundary conditions that uniquely determine the initial and final configuration of the manipulator are determined by the criterion of minimal discomfort (Marler et al., 2005) 


$$
K_{2}=\frac{1}{G} \sum_{i=1}^{n}\left[\gamma_{i}\left(\Delta q_{i}^{\text {nom }}\right)^{2}+G \times Q U_{i}+G \times Q L_{i}\right] \rightarrow \min ,
$$

where $Q U_{i}=\left[0.5 \sin \left(\frac{5.0\left(q_{i}^{U}-q_{i}\right)}{q_{i}^{U}-q_{i}^{L}}+\frac{\pi}{2}\right)+1\right]^{100}$,

$$
Q L_{i}=\left[0.5 \sin \left(\frac{5.0\left(q_{i}-q_{i}^{L}\right)}{q_{i}^{U}-q_{i}^{L}}+\frac{\pi}{2}\right)+1\right]^{100}, \Delta q_{i}^{\text {norm }}=\frac{q_{i}-q_{i}^{N}}{q_{i}^{U}-q_{i}^{L}},
$$

$q_{i}, q_{i}^{N}, q_{i}^{U}, q_{i}^{L}-$ current, neutral, maximum and minimum generalized coordinate values, respectively; $\gamma_{i}-$ weight coefficients and $G$ - some constant.

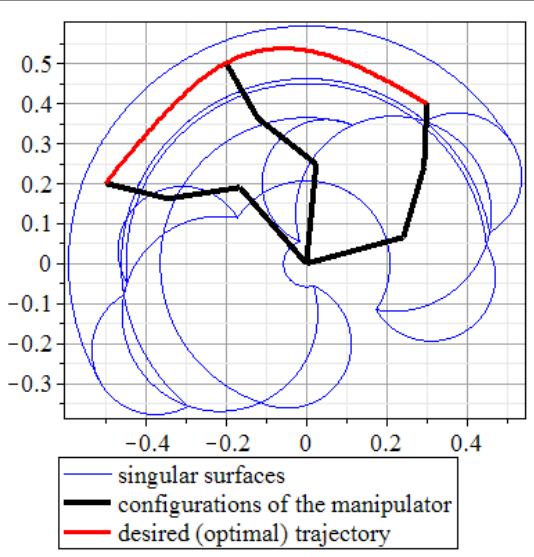

Fig. 1. One of the variants of the planned trajectories of the manipulator end-effector in accordance with a set of criteria (2) and (5)

Fig. 1 shows one of the optimal (in accordance with the criteria (2) and (4)) trajectories, passing through three given points of the workspace.

\section{CONTROL SYSTEM}

In accordance with (Seraji, 1987) the total control signal needed to track the optimal trajectory, consists of three components

$$
u(t)=u_{f f}(t)+u_{f b}(t)+u_{a x}(t),
$$

where $u_{f f}(t)$ - signal generated by the feed forward controller, which uses the inversion of the manipulator; $u_{f b}(t)$ - signal generated by the feedback controller at the operating point $P_{j}$; $u_{a x}(t)$ - additional signal generated in accordance with the parameters of the operating point $P_{j}=\left\{T\left(t_{j}\right), \Phi\left(t_{j}\right), \dot{\Phi}\left(t_{j}\right)\right\}$ at a certain time movement $t_{j}$.

Complete manipulator control law has the following form

$$
\begin{aligned}
& T(t)=\tau_{f f}(t)+\tau_{f b}(t)+\tau\left(t_{j}\right)= \\
& =A \ddot{\theta}_{d}(t)+B \dot{\theta}_{d}(t)+C \theta_{d}(t)+K_{v} \dot{e}(t)+K_{p} e(t)+\tau\left(t_{j}\right),
\end{aligned}
$$

where $\theta_{d}(t)=\Phi_{d}(t)-\Phi\left(t_{j}\right)$ - disturbance of the manipulator configuration in the neighborhood of the operating point; $A=\left.\frac{\partial L(\Phi, \dot{\Phi}, \ddot{\Phi})}{\partial \ddot{\Phi}}\right|_{P_{j}} ; B=\left.\frac{\partial L(\Phi, \dot{\Phi}, \ddot{\Phi})}{\partial \dot{\Phi}}\right|_{P_{j}} ; C=\left.\frac{\partial L(\Phi, \dot{\Phi}, \ddot{\Phi})}{\partial \Phi}\right|_{P_{j}}-$ the coefficients of Taylor series of the left side $L(\Phi, \dot{\Phi}, \ddot{\Phi})$ of the equation (1); $e(t)=\theta_{d}(t)-\theta(t)$ - error column vector of the position tracking; $K_{p}$ and $K_{v}$ - matrices of the feedback coefficients of position and velocity respectively.

In (Seraji, 1987) it is showed, that $e(t)$ can be represented in the form of $n$ isolated second order differential equations of the form

$$
\ddot{e}(t)+D_{1} \dot{e}(t)+D_{2} e(t)=0,
$$

where $D_{1}=\operatorname{diag}\left\{2 \xi_{i} \omega_{i}\right\} ; D_{2}=\operatorname{diag}\left\{\omega_{i}^{2}\right\} ; \quad \xi_{i}, \omega_{i}, i=1, \ldots, n$ previously taken by the designer damping factors and eigen frequencies tracking error, respectively. Then $K_{v}=A D_{1}-B$ and $K_{p}=A D_{2}-C$.

This control system can be implemented enough simply in practice.

\section{CONCLUSION}

Simulation of the manipulator end-effector movement on optimal trajectories was performed in two ways: by using a Cosirop program, supplied with the robot Mitsubishi RV-1A and internal control systems, as well as with the adaptive control system described above.

Simulation results demonstrates efficiency in tracking of optimal trajectories of the end-effector. By choosing the appropriate values $\xi_{i}, \omega_{i}$, can be obtain the minimum value of the tracking error vector at different end-effector velocities

Thus, the proposed approach allows us to generate and implement trajectories are optimal from the perspective of different quality criteria, including biomechanical criteria. Such control system is planned to be used in control systems of a prosthetic limbs with a four-bar heterogeneous mechanism of the knee.

\section{REFERENCES}

LaValle, S. M. ((2006). Planning Algorithms, Cambridge University Press, ISBN 0-52-186205-1, New York, USA

Engelbrecht, S.E. (2001). Minimum Principles in Motor Control.Journal ofMathematical Psychology, Vol. 45, No. 3, (August 2001), pp. 497-542,ISSN: 0022-2496

Uno, Y.; Kawato, M. \& Suzuki, R. (1989). Formation andControl of Optimal Trajectory in Human Multijoint Arm Movement: Minimum Torque-Change Model. Biological Cybernetics, Vol. 61, No. 2, (June 1989), pp. 89-101, ISSN: 1432-0770

Nakano, E.; Imamizu, H.; Osu, R.; Uno, Y.; Gomi, H.; Yoshioka, T. \& Kawato, M. (1999).Quantitative Examinations of Internal Representationsfor Arm Trajectory Planning: Minimum CommandedTorque Change Model. Journal of Neurophysiology, Vol. 81, No. 5, (May 1999), pp. 2140-2155, ISSN: $1522-1598$

Crevecoeur, F.; Thonnard, J.-L. \& Lefe`vre, P. (2009). Optimal Integration of Gravity in Trajectory Planning of Vertical Pointing Movements. Journal of Neurophysiology, Vol. 102, No. 2, (August2009), pp. 786-796, ISSN: 1522-1598

Marler, R.T.; Rahnatalla, S.; Shanahan, M. \& Abdel-Malek, K. (2005). A new discomfort function for optimization-based posture prediction. SAE Human modeling for design and engineering conference, ISSN 0148-7191, 2006-01-2334 SAE technical paper series, Iowa city, USA

Seraji, H. (1987). An Approach to Multivariable Control of Manipulators. Journal of dynamic systems, measurement and control. Vol. 109, No. 2, (February 1987), pp. 146-154, ISSN: 0022-0434 